\title{
Spatial Resolution and Sensitivity of the Inveon Small-Animal PET Scanner
}

Eric P. Visser, Jonathan A. Disselhorst, Maarten Brom, Peter Laverman, Martin Gotthardt, Wim J.G. Oyen, and Otto C. Boerman

Department of Nuclear Medicine, Radboud University Nijmegen Medical Centre, Nijmegen, The Netherlands

The Inveon small-animal PET scanner is characterized by a large, 127-mm axial length and a 161-mm crystal ring diameter. The associated high sensitivity is obtained by using all lines of response (LORs) up to the maximum ring difference (MRD) of 79, for which the most oblique LORs form acceptance angles of $38.3^{\circ}$ with transaxial planes. The result is 2 phenomena that are normally not encountered in PET scanners: a parallax or depth-of-interaction effect in the axial direction and the breakdown of Fourier rebinning (FORE). Both effects cause a deterioration of axial spatial resolution. Limiting the MRD to smaller values reduces this axial blurring at the cost of sensitivity. Alternatively, 3-dimensional (3D) reconstruction techniques can be used in which the rebinning step is absent. The aim of this study was to experimentally determine the spatial resolution and sensitivity of the Inveon for its whole field of view (FOV). Methods: Spatial resolution and sensitivity were measured using filtered backprojection (FBP) with FORE, FBP with LOR angle-weighted adapted FORE (AFORE), and $3 D$ ordered-subset expectation maximization followed by maximum a posteriori reconstruction (OSEM3D/ MAP). Results: Tangential and radial full width at half maximum (FWHM) showed almost no dependence on the MRD using FORE and FBP. Tangential FWHMs were $1.5 \mathrm{~mm}$ in the center of the FOV (CFOV) and $1.8 \mathrm{~mm}$ at the edge of the FOV (EFOV). Radial FWHMs were 1.5 and $3.0 \mathrm{~mm}$ in the CFOV and EFOV, respectively. In contrast, axial FWHMs increased with the MRD and ranged between 1.1 and $2.0 \mathrm{~mm}$ in the CFOV and between 1.5 and $2.7 \mathrm{~mm}$ in the EFOV for a MRD between 1 and 79. AFORE improved the axial resolution for a large part of the FOV, but image noise increased. OSEM3D/MAP yielded uniform spatial resolution in all directions, with an average FWHM of $1.65 \pm 0.06 \mathrm{~mm}$. Sensitivity in the CFOV for the default energy and coincidence time window was 0.068 ; peak sensitivity was 0.111 . Conclusion: The Inveon showed high spatial resolution and high sensitivity, both of which can be maintained using OSEM3D/MAP reconstruction instead of rebinning and 2D algorithms.

Key Words: positron emission tomography; small-animal; spatial resolution; sensitivity; image reconstruction

J Nucl Med 2009; 50:139-147

DOI: 10.2967/jnumed.108.055152

Received Jun. 20, 2008; revision accepted Oct. 6, 2008.

For correspondence or reprints contact: Eric P. Visser, Department of Nuclear Medicine (Internal Postal Code 444), Radboud University Nijmegen Medical Centre, P.O. Box 9101, 6500 HB Nijmegen, The Netherlands.

E-mail: e.visser@nucmed.umcn.nl

COPYRIGHT ๑ 2009 by the Society of Nuclear Medicine, Inc.
$\mathbf{T}$ he Inveon (Siemens) small-animal PET scanner differs from its predecessor, the microPET Focus 120 (F120; Siemens), by its larger lutetium orthosilicate (LSO) detector blocks, improved processing of high-speed events $(1-4)$, and shorter, tapered light guides coupling the detector blocks to the photomultiplier tubes (5). The Inveon contains 4 rings of 16 blocks-20 $\times 20$ detectors of $1.5 \times 1.5 \mathrm{~mm}$ each, resulting in an axial length of $127 \mathrm{~mm}$. The F120 consists of 4 rings of 24 blocks $-12 \times 12$ detectors of the same size, leading to an axial length of $76 \mathrm{~mm}$. Table 1 compares the geometric properties of the Inveon with several other commercial crystal-based small-animal PET scanners.

The main advantage of the larger axial field of view (FOV) of the Inveon is the higher detection efficiency (or sensitivity), with a peak value as specified by the manufacturer of greater than 0.1 in the center of the FOV (CFOV) for the maximum width of the energy window. However, high sensitivity is obtained only when using all lines of response (LOR), up to the maximum ring difference (MRD) of 79. With its crystal ring diameter of $161 \mathrm{~mm}$ and axial length of $127 \mathrm{~mm}$ (aspect ratio, 0.79), the most oblique LORs form acceptance angles of $38.3^{\circ}$ with the transaxial planes, leading to 2 phenomena that are normally not encountered in PET scanners. First, the parallax or depth-of-interaction (DOI) effect due to crystal penetration of oblique LORs in the axial direction cannot be ignored, and second, conventional Fourier rebinning (FORE) for 2-dimensional (2D) reconstruction techniques (6) breaks down. Both effects lead to a deterioration of the axial spatial resolution. Parallax or DOI effects are well known in PET scanners but have been described and measured mainly for the radial resolution at radial offsets from the CFOV (7) and not for the axial resolution. The performance of FORE for large acceptance angles has been reported by Matej et al. (8) for angles up to $26.2^{\circ}$. A considerable deterioration of the axial resolution for large acceptance angles, especially at large radial offsets, was found in that study.

The advantage of using FORE with 2D reconstruction techniques such as filtered backprojection (FBP) or 2D ordered-subset expectation maximization (OSEM2D) is high processing speed. FORE has, therefore, been imple- 
TABLE 1. Geometric Properties of Several Commercial, Crystal-Based Small-Animal PET Scanners

\begin{tabular}{|c|c|c|c|c|c|c|c|}
\hline $\begin{array}{c}\text { PET } \\
\text { scanner }\end{array}$ & $\begin{array}{c}\text { Crystal } \\
\text { element } \\
\text { size }\left(\mathrm{mm}^{3}\right)\end{array}$ & $\begin{array}{l}\text { No. of } \\
\text { crystal } \\
\text { rings }\end{array}$ & $\begin{array}{l}\text { Detector } \\
\text { material }\end{array}$ & $\begin{array}{c}\text { Axial } \\
\text { length }(\mathrm{mm})\end{array}$ & $\begin{array}{l}\text { Crystal ring } \\
\text { diameter } \\
(\mathrm{mm})\end{array}$ & $\begin{array}{l}\text { Aspect } \\
\text { ratio* }\end{array}$ & $\begin{array}{c}\text { Largest LOR } \\
\text { acceptance } \\
\text { angle }^{\dagger} \text { (degrees) }\end{array}$ \\
\hline Inveon & $1.5 \times 1.5 \times 10$ & 80 & LSO & 127 & 161 & 0.79 & 38.3 \\
\hline $\mathrm{F} 120(12)$ & $1.5 \times 1.5 \times 10$ & 48 & LSO & 76 & 147 & 0.52 & 27.3 \\
\hline Mosaic (13) & $2 \times 2 \times 10$ & 52 & GSO & 119 & 197 & 0.60 & $28.0^{\ddagger}$ \\
\hline Vista (14) & $1.45 \times 1.45 \times(8+7)$ & 26 & LYSO/GSO phoswich & 48 & 118 & 0.41 & 22.1 \\
\hline ClearPET $(15,16)$ & $2 \times 2 \times(10+10)$ & 32 & LYSO/LuYAP phoswich & 110 & 135 & 0.81 & 39.2 \\
\hline $\begin{array}{l}{ }^{*} \text { Aspect ratio is c } \\
{ }^{\dagger} \text { LOR acceptance } \\
\text { ”This LOR angle } \\
\text { GSO = gadoliniu }\end{array}$ & $\begin{array}{l}\text { ystal ring diameter divic } \\
\text { angle is angle between } \\
\text { determined by softwa } \\
\text { oxyorthosilicate; LYS }\end{array}$ & $\begin{array}{l}\text { d by a) } \\
\text { OR an } \\
\text {; LOR } \\
=\text { lutet }\end{array}$ & $\begin{array}{l}\text { length. } \\
\text { transaxial planes. } \\
\text { gle from aspect ratio } \\
\text { m yttrium orthosilicate; }\end{array}$ & $\begin{array}{l}\text { ld be som } \\
\text { YAP = lut }\end{array}$ & $\begin{array}{l}\text { at larger. } \\
\text { m yttrium }\end{array}$ & กinu & ovskit \\
\hline
\end{tabular}

mented in most standard software packages for both clinical and preclinical PET scanners. The deterioration of axial resolution by FORE could be avoided by using 3-dimensional (3D) reconstruction techniques in which the rebinning step is not present and all LORs contained in the 3D sinograms are directly used in the image reconstruction. Although algorithms such as 3D OSEM (OSEM3D), maximum a posteriori reconstruction (MAP), and 3D reprojection (3DRP) are available on some (preclinical) scanners, the use of FORE and 2D reconstruction is still widespread. This is mainly due to the higher complexity and long reconstruction times of the $3 \mathrm{D}$ algorithms, which can take orders of magnitude more time than 2D algorithms, including the time for the rebinning. Especially in routine clinical settings, longer reconstruction times can be a prohibitive factor for using 3D reconstruction algorithms. Moreover, because of requirements about the minimum patient port size in clinical scanners, aspect ratios are generally not large and errors due to parallax or DOI effects and FORE will, in general, be smaller than those in small-animal scanners.

A different, more practical way to improve the axial resolution while still using FORE and 2D reconstruction is to decrease the maximum LOR acceptance angle by reducing the MRD down to a level at which FORE becomes more accurate. This, however, reduces the effective sensitivity of the scanner, because fewer LORs and, thus, fewer measured coincidences are included in the rebinning and reconstruction process. On the other hand, the sensitivity will become more uniform across the axial FOV. This method is also applied in clinical, whole-body PET scanners, in which bed overlap issues may be important. The Biograph TrueV PET/CT scanner (Siemens), for instance, uses as a default MRD of 38, with the number of crystal rings equaling 52. Similar choices have been made for the older Biograph types of scanners and the ECAT EXACT (Siemens).

The aim of this study was to experimentally determine the spatial resolution and sensitivity of the Inveon for its whole FOV. The standard reconstruction and rebinning were FBP and FORE. A comparison was made with a new, adapted FORE (AFORE) algorithm recently incorporated in the standard scanner software. This algorithm uses LOR angle-weighted FORE in such a way that coincidence counts belonging to large LOR acceptance angles are rebinned with reduced weighting factors. Detailed information about this algorithm was not provided by the manufacturer. Further, a combination of OSEM3D and MAP was used for 2 different settings of the MAP prior. The results were compared with those obtained by the $2 \mathrm{D}$ reconstruction techniques.

Finally, the quality of images of real animals also depends on parameters such as scatter fraction and countingrate performance. Assessment of overall image quality with these parameters considered, however, was outside the scope of this study.

\section{MATERIALS AND METHODS}

Spatial resolution and sensitivity were measured using a ${ }^{22} \mathrm{Na}$ point source with an active diameter of less than $0.25 \mathrm{~mm}$ embedded in a Lucite disk (Isotope Products Laboratories [IPL]). The activity of the point source was $0.918 \mathrm{MBq}$, as measured in a dose calibrator (VDC-404; Veenstra) in our laboratory. This value was corrected for the point source geometry because the device had been calibrated for extended sources (syringes). The accuracy of the activity is $\pm 4 \%$, which results from $\pm 3 \%$ as specified by the manufacturer and an additional $\pm 1 \%$ to account for uncertainties in the point source geometry correction.

Spatial-resolution measurements were not corrected for source dimension, positron range, or noncolinearity of the 2 photons involved in positron annihilation. The point source was placed in the FOV of the scanner for transaxial positions ranging from 0 to $46 \mathrm{~mm}$ from the center and from 0 to $7 \mathrm{~mm}$ in the opposite direction. This range was determined by the vertical bed-motion limitations of the scanner. The axial positions covered a total range of $200 \mathrm{~mm}$ around the CFOV. This large axial range also was used to record coincidence counts for source positions outside the axial FOV. The transaxial step size was $1 \mathrm{~mm}$ for the range of -7 to $12 \mathrm{~mm}$ and $2 \mathrm{~mm}$ for the range of $12-46 \mathrm{~mm}$. The axial step size was $0.80 \mathrm{~mm}$ (approximate thickness of 1 transaxial plane) for the 
range of -8 to $8 \mathrm{~mm}$ and $4.78 \mathrm{~mm}$ ( 6 transaxial planes) elsewhere. All measurements along the transaxial direction were repeated 4 times, with the point source stepped in the axial direction (step size, $0.2 \mathrm{~mm}$ ) to obtain oversampled axial profiles by interleaving. For each position of the point source, 10 million coincidence counts were collected.

Spatial resolution was determined using FORE and FBP with a ramp filter at the Nyquist frequency. The pixel size in transaxial planes was $0.194 \mathrm{~mm}$, and the plane separation was $0.796 \mathrm{~mm}$. The image matrix size was $512 \times 512 \times 159$. Profiles through count-distribution peaks were drawn in these orthogonal directions for a middle plane to determine transaxial radial and tangential resolutions at each point source position. According to the National Electrical Manufacturers Association (NEMA) requirements (NEMA-NU2-2007 (9)), the full width at half maximum (FWHM) and full width at tenth maximum (FWTM) were determined by linear interpolation between adjacent pixels at half or one tenth of the profile maximum value, which was determined by a parabolic fit using the peak point and its 2 nearest neighbors. Axial resolutions were obtained in the same way using the interleaved profiles.

Before reconstruction, the list-mode data were histogrammed with a span of 3 and MRDs of 79, 46, 25, 13, and 1 into 3D sinograms. The maximum acceptance angles corresponding to these MRDs are $38.3^{\circ}, 24.9^{\circ}, 14.4^{\circ}, 7.9^{\circ}$, and $1.1^{\circ}$, respectively. An MRD of 46 was specifically chosen for a sensitivity comparison with the F120, which is geometrically similar except for the smaller axial length and number of crystals per detector block. In principle, for a direct one-to-one comparison, an MRD of 47 should be chosen in accordance with the 48 detector rings in the F120. However, the closest attainable setting in the Inveon is 46, because for complete segments in the 3D sinograms, MRD is restricted by $\mathrm{MRD}=(n+1 / 2) \times \operatorname{span}-1 / 2(10)$.

In addition, spatial resolution was determined using AFORE, characterized by its LOR angle-based weighting factors. Spatial resolution was determined for the same point source positions and NEMA procedure as described above, again using FBP with the same pixel and image matrix sizes.

For a limited number of point source positions in the radial direction, data were reconstructed using OSEM3D/MAP (2 OSEM3D iterations and 18 MAP iterations). The MAP reconstructions were performed with $\beta=0.5$ and 1.5 and were optimized for uniform resolution. The transaxial pixel size was $0.331 \mathrm{~mm}$, and the image matrix size was $256 \times 256 \times 159$. The same settings as for FBP (transaxial pixel size, $0.194 \mathrm{~mm}$; matrix size, $512 \times 512 \times 159$ ) were not possible because of the limited number of P-matrices and blur kernels available for these 3D reconstruction algorithms. However, the transaxial pixel size of $0.331 \mathrm{~mm}$ was still small enough to determine tangential and radial resolutions accurately (NEMA requires the pixel size to be no more than one third of the expected FWHM, which is clearly fulfilled).

The axial resolutions, on the contrary, were determined in the same way as for FBP, using the $0.2-\mathrm{mm}$ interleaved profiles. The reason for limiting the number of point source positions was the long reconstruction time for OSEM3D/MAP. One reconstruction using the above-mentioned matrix size and iterations setting took approximately $4 \mathrm{~h}$ on a personal computer (Dell), with 16GB RAM and a Dual-Core Xeon 5160 processor (Intel) running at $3 \mathrm{GHz}$ under Windows XP (64 bit; Microsoft). Because of the axial interleaving, each reconstruction was performed 4 times, leading to a total reconstruction time of $16 \mathrm{~h}$ per point source position.
The coincidence timing window $(\Delta \mathrm{t})$ and the energy window $(\Delta \mathrm{E})$ were used at their default settings of $3.4 \mathrm{~ns}$ and $350-650 \mathrm{keV}$, respectively. For the CFOV only, additional measurements with a $\Delta \mathrm{E}$ of $250-750 \mathrm{keV}$ and a $\Delta \mathrm{t}$ of $2.8,4.1$, and $4.7 \mathrm{~ns}$ were performed. Randoms were subtracted using a delayed-window technique. Dead-time correction was based on a global singles-rate estimate.

The sensitivity of the scanner was calculated as the ratio of the histogrammed trues rate and the rate at which photon pairs are emitted from the point source. The latter was determined using the point source activity, taking into account the positron branching ratio of 0.899 for ${ }^{22} \mathrm{Na}(11)$. Sensitivity data were generated for all point source positions as specified above. Furthermore, to cover the complete FOV, additional radial sensitivity profiles with radial steps of $5 \mathrm{~mm}$ were recorded for an MRD of 79 in transaxial planes that were axially separated by $10 \mathrm{~mm}$.

Because of its LOR angle-dependent weighting factors that reduce the contribution of oblique LORs to the reconstructed image, it might be expected that AFORE be associated with lower effective sensitivities than FORE. Because the histogrammed trues rate is the same for both rebinning algorithms, a comparison can only be made on the basis of noise levels in reconstructed images. For this purpose, we compared the noise levels in images of a homogeneous ${ }^{68} \mathrm{Ge}$ cylinder using FORE and AFORE with FBP and a transaxial matrix size of $128 \times 128$.

Finally, IAW 1.0.2 (Inveon Acquisition Workplace; Siemens) was used for the FORE and FBP results, and IAW 1.0.4 was used for the AFORE/FBP and OSEM3D/MAP results. The return to version 1.0.2 was necessary because the FORE algorithm was not available anymore in version 1.0.4. The FBP algorithms were exactly the same in both versions.

\section{RESULTS}

\section{Spatial Resolution}

The results for transaxial tangential and radial resolutions using FORE and FBP are shown in Figure 1A for an MRD of 79. These transaxial resolutions were found to be almost independent of MRD; therefore, the results for the other MRDs have been left out for clarity. The axial resolutions, on the contrary, were highly dependent on the MRD and increased as a function of radial distance from the CFOV, as shown in Figure 1B.

According to NEMA-NU2-2007 (9), which was designed for the characterization of clinical PET scanners, spatial resolution should be reported for source positions at radial offsets of 1 and $10 \mathrm{~cm}$ to obtain numbers that can be considered representative for the CFOV and the edge of the FOV (EFOV), respectively. For small-animal scanners, NEMA prescriptions do not exist. We chose to average the resolution values for radial offsets between 2 and $5 \mathrm{~mm}$, yielding representative numbers for the CFOV (averaged over 8 point source positions), and between 36 and $46 \mathrm{~mm}$, representing the radial EFOV (averaged over 6 point source positions). The resulting FWHMs have been summarized in Table 2.

Spatial resolutions in all 3 directions were found to be independent of the MRD using AFORE and FBP. A comparison of the AFORE and FORE results for the transaxial resolutions is shown in Figure 2A. Only the results for an 


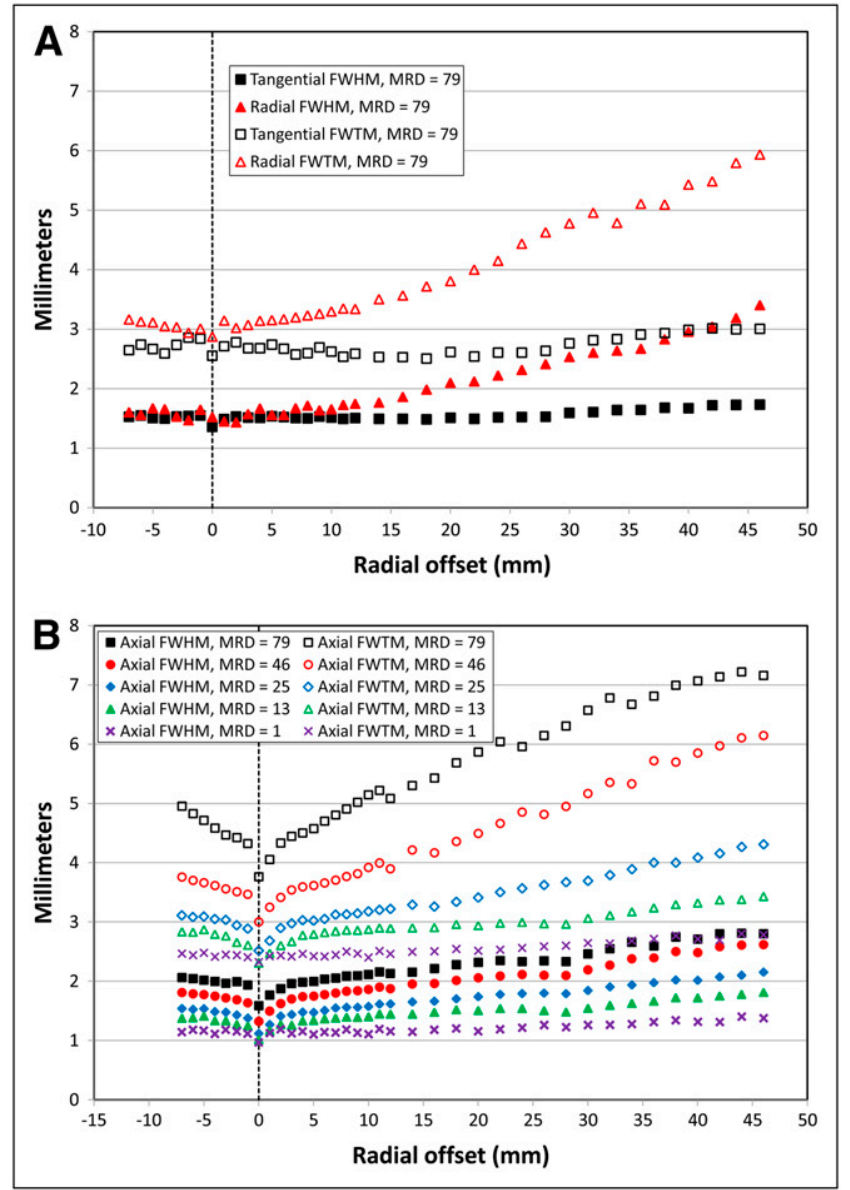

FIGURE 1. (A) Transaxial spatial resolutions (FWHM and FWTM) obtained with FORE and FBP, using MRD of 79 as function of radial distance from CFOV. Other settings for MRD yielded highly similar results, which have been left out for clarity. (B) Axial spatial resolutions (FWHM and FWTM) obtained with FORE and FBP as function of radial distance from CFOV for different MRD settings.

MRD of 79 have been plotted, because both algorithms yielded negligible differences on variation of MRD. A comparison of the axial resolutions is shown in Figure 2B. For AFORE, only the results for an MRD of 79 have been plotted because differences upon variation of MRD were negligible. However, the FORE results depended strongly on the MRD, as already shown in Figure 1A. Therefore, as a typical example for smaller MRDs, the graph for FORE with an MRD of 13 is included in Figure 2B. The averaged FWHMs for CFOV and EFOV obtained with AFORE are presented in Table 2.

The radial profiles of FWHM and FWTM in all directions for OSEM3D/MAP for both MAP smoothing parameters $(\beta=0.5$ and 1.5) are shown in Figures $3 \mathrm{~A}$ and $3 \mathrm{~B}$. The numeric values of FWHM for CFOV and EFOV are given in Table 2.

\section{Sensitivity}

Figures $4 \mathrm{~A}$ and $4 \mathrm{~B}$ show axial and radial profiles, respectively, of sensitivity using default $\Delta \mathrm{E}$ and $\Delta \mathrm{t}$ for different
MRDs. The axial profiles are for the radial center of the scanner; the radial profiles are for a middle plane. Point source positions were as indicated in the "Materials and Methods" section of this article.

Figure 4C shows the sensitivity obtained for the whole FOV for an MRD of 79 using the additional radial profiles separated by $10 \mathrm{~mm}$ in the axial direction. On the basis of cylinder symmetry, the original data were mirrored 2 -fold to cover the complete FOV.

The effect of different $\Delta \mathrm{E}$ and $\Delta \mathrm{t}$ settings on the sensitivity in the CFOV is shown in Table 3. This table also shows the sensitivity as calculated from a total trues rate instead of from a histogrammed trues rate (numbers in parentheses). The corresponding spatial resolutions were found to be essentially independent of $\Delta \mathrm{E}$ and $\Delta \mathrm{t}$.

The images of the homogeneous ${ }^{68} \mathrm{Ge}$ cylinder that were used to compare the noise levels associated with FORE and AFORE are shown in Figure 5. As an indication for image noise, we used the relative $\mathrm{SD}\left(\mathrm{SD}_{\text {rel }}=\mathrm{SD}\right.$ divided by mean pixel value) in a central circular region of interest of $15-\mathrm{mm}$ diameter, as shown in the figure. The resulting values for $\mathrm{SD}_{\text {rel }}$ were 0.10 and 0.22 for FORE and AFORE, respectively, indicating that FORE resulted in lower noise levels.

\section{DISCUSSION}

\section{Spatial Resolution}

To compare spatial resolutions of different PET scanners, an agreement on rebinning and reconstruction algorithms has to be made. According to NEMA-NU2-2007 (9), the reconstruction method should be FBP. Although no prescription exists for the rebinning method, in most cases FORE is used, as was done in the present study.

In Figure 1A, the deterioration of the transaxial radial resolution at the EFOV is clearly noticed. The transaxial tangential resolution, on the contrary, is more or less constant over the complete radial range of point source positions. We observed only a negligible dependency of both transaxial resolutions on MRD. These observations are in line with results for other (clinical and preclinical) PET scanners, for which the deterioration of the radial resolution has been attributed to the transaxial parallax or DOI effect (7).

As can be seen in Figure 1B and Table 2, axial resolution is markedly deteriorated for large MRDs. Both the axial parallax or DOI effect and the breakdown of FORE contribute to this deterioration. These effects are caused by large LOR acceptance angles, but whereas the breakdown of FORE is a mathematic phenomenon, the axial parallax or DOI effect is related to the way in which coincidence photons are being detected in the crystal elements. In general, these effects cannot be disentangled. However, for the radial CFOV, FORE is exact, and the larger FWHM should be attributed entirely to the axial parallax or DOI effect. In the CFOV for an MRD of 79, axial FWHM is $1.98 \mathrm{~mm}$, and by comparison with an MRD of 1, for which axial FWHM 
TABLE 2. Spatial Resolution (FWHM) for CFOV and Radial EFOV

\begin{tabular}{|c|c|c|c|c|c|c|}
\hline \multirow[b]{2}{*}{ Method of measurement } & \multicolumn{3}{|c|}{$\mathrm{CFOV}^{*}$} & \multicolumn{3}{|c|}{ Radial EFOV ${ }^{\dagger}$} \\
\hline & Tangential & Radial & Axial & Tangential & Radial & Axial \\
\hline \multicolumn{7}{|l|}{ FORE, FBP } \\
\hline $\mathrm{MRD}=79$ & $1.52 \pm 0.02$ & $1.57 \pm 0.09$ & $1.98 \pm 0.04$ & $1.70 \pm 0.04$ & $3.02 \pm 0.26$ & $2.75 \pm 0.08$ \\
\hline $\mathrm{MRD}=46$ & $1.52 \pm 0.02$ & $1.56 \pm 0.09$ & $1.72 \pm 0.05$ & $1.74 \pm 0.04$ & $2.99 \pm 0.23$ & $2.53 \pm 0.09$ \\
\hline $\mathrm{MRD}=25$ & $1.52 \pm 0.02$ & $1.54 \pm 0.09$ & $1.47 \pm 0.04$ & $1.74 \pm 0.04$ & $2.95 \pm 0.17$ & $2.06 \pm 0.06$ \\
\hline $\mathrm{MRD}=13$ & $1.51 \pm 0.02$ & $1.52 \pm 0.08$ & $1.32 \pm 0.05$ & $1.78 \pm 0.04$ & $2.95 \pm 0.21$ & $1.75 \pm 0.05$ \\
\hline $\mathrm{MRD}=1$ & $1.47 \pm 0.02$ & $1.51 \pm 0.08$ & $1.14 \pm 0.03$ & $1.83 \pm 0.07$ & $2.94 \pm 0.20$ & $1.51 \pm 0.07$ \\
\hline \multicolumn{7}{|l|}{ AFORE, FBP } \\
\hline $\mathrm{MRD}=79$ & $1.50 \pm 0.02$ & $1.51 \pm 0.08$ & $1.30 \pm 0.05$ & $2.12 \pm 0.07$ & $2.80 \pm 0.23$ & $3.17 \pm 0.40$ \\
\hline \multicolumn{7}{|l|}{ OSEM3D/MAP } \\
\hline$\beta=1.5 \mathrm{~mm}$ & $1.69 \pm 0.04$ & $1.68 \pm 0.02$ & $1.71 \pm 0.03$ & 1.85 & 1.99 & 1.68 \\
\hline$\beta=0.5 \mathrm{~mm}$ & $1.57 \pm 0.04$ & $1.56 \pm 0.02$ & $1.66 \pm 0.02$ & 1.70 & 1.82 & 1.62 \\
\hline \multicolumn{7}{|c|}{$\begin{array}{l}{ }^{*} \text { Values for CFOV for 2D reconstructions were obtained by averaging over } 8 \text { point source positions }(2-5 \mathrm{~mm} \text { from radial center). For } \\
\text { OSEM3D/MAP, averaging was done for } 2 \text { positions }(2 \text { and } 6 \mathrm{~mm} \text { from radial center). } \\
{ }^{\dagger} \text { Values for radial EFOV for } 2 \mathrm{D} \text { reconstructions were obtained by averaging over } 6 \text { point source positions ( } 36-46 \mathrm{~mm} \text { from radial center). } \\
\text { Cor OSEM3D/MAP, value at } 36 \mathrm{~mm} \text { from radial center was taken. } \\
\text { Errors are SD belonging to averaging as indicated. }\end{array}$} \\
\hline
\end{tabular}

is $1.14 \mathrm{~mm}$, it can be concluded that approximately $0.8 \mathrm{~mm}$ of increase of axial FWHM is caused by the parallax or DOI effect. For example, for the crystal size of $1.5 \times 1.5 \times 10 \mathrm{~mm}$ and a pitch of $1.59 \mathrm{~mm}$, the absorption length will be distributed axially over 5 neighboring crystals when using a 10 -mm crystal length as the typical length over which detected photons are absorbed, for photons with the maximum LOR acceptance angle of $38.3^{\circ}$. At the EFOV, also the breakdown of FORE adds to the deterioration of axial resolution, as shown by an axial FWHM of $2.75 \mathrm{~mm}$ for an MRD of 79 .

For the smallest MRDs, the axial resolution is significantly better than the transaxial ones, both for the radial CFOV and for the EFOV. For an MRD of 1 (corresponding to a $2 \mathrm{D}$ mode operation of the scanner), we even found a submillimeter axial FWHM of $0.97 \mathrm{~mm}$ (Fig. 1B, value at exact CFOV). This FWHM value could be explained as follows. For LORs passing through the center of the FOV striking both detector elements perpendicularly, the theoretic FWHM - determined on the basis of only geometric arguments—equals $d / 2(d=$ crystal size $)$ if the point source is located midway between both detectors and approaches $d$ when the source is close to 1 of the detectors (7). However, because the crystal elements are arranged in blocks with flat faces, they are not located on an exact circle. LORs passing through the CFOV strike the outermost crystals of a detector block under an angle of approximately $5^{\circ}$ with the normal in the transaxial direction. Therefore, a certain parallax or DOI effect will be present in transaxial directions, even if the point source is located at the exact CFOV. The axial resolution, on the contrary, is not influenced, because cylinder symmetry implies that axially, the photons strike all crystal elements perpendicularly provided MRD is small. Finally, the fact that we do not observe an axial FWHM of exactly $d / 2=0.75 \mathrm{~mm}$ may be attributed to a combination of many confounding effects: noncoli- nearity of the photon pairs, finite source dimension, finite positron range, geometric imperfections in the detector block array, or intracrystal scattering.

To fully exploit the high sensitivity of the Inveon, it is clear from Figure 4A that an MRD of 79 should be used. However, it was demonstrated that for $2 \mathrm{D}$ reconstruction and FORE, a serious deterioration of axial resolution occurs. The AFORE algorithm overcomes this problem of deterioration to a certain extent. As can be seen in Figure $2 \mathrm{~B}$, the axial resolution is indeed considerably improved for an MRD of 79 when using AFORE instead of FORE. However, for small MRDs there is no improvement, and in the EFOV axial resolution is even seriously deteriorated using AFORE, as can be seen in Figure 2B in the comparison with FORE at an MRD of 13. Furthermore, although we expected that AFORE would not influence transaxial resolutions, Figure 2A demonstrates that already for radial offsets greater than $15 \mathrm{~mm}$, the tangential FWHM is larger for AFORE than it is for FORE. This effect is even stronger for FWTM, which is associated with highly nongaussian tangential profiles with extending tails for AFORE. Because no information is available on the mathematics used in AFORE, we presently cannot explain these phenomena. Finally, because AFORE results in lower effective sensitivity, as demonstrated by Figure 5 and the corresponding $\mathrm{SD}_{\text {rel }}$ values, we believe that AFORE is not the reconstruction method of choice for the Inveon.

For users who are not prohibited by the long reconstruction times of OSEM3D/MAP ( $4 \mathrm{~h}$ for $256 \times 256$ and $1 \mathrm{~h}$ for $128 \times 128$ image planes on the personal computer configuration, as mentioned in "Materials and Methods"), this reconstruction method is preferable in terms of uniform, high resolution and high sensitivity. As can be seen in Figures $3 \mathrm{~A}$ and $3 \mathrm{~B}$, the spatial resolution is uniform across the FOV of the scanner and almost direction-independent, 

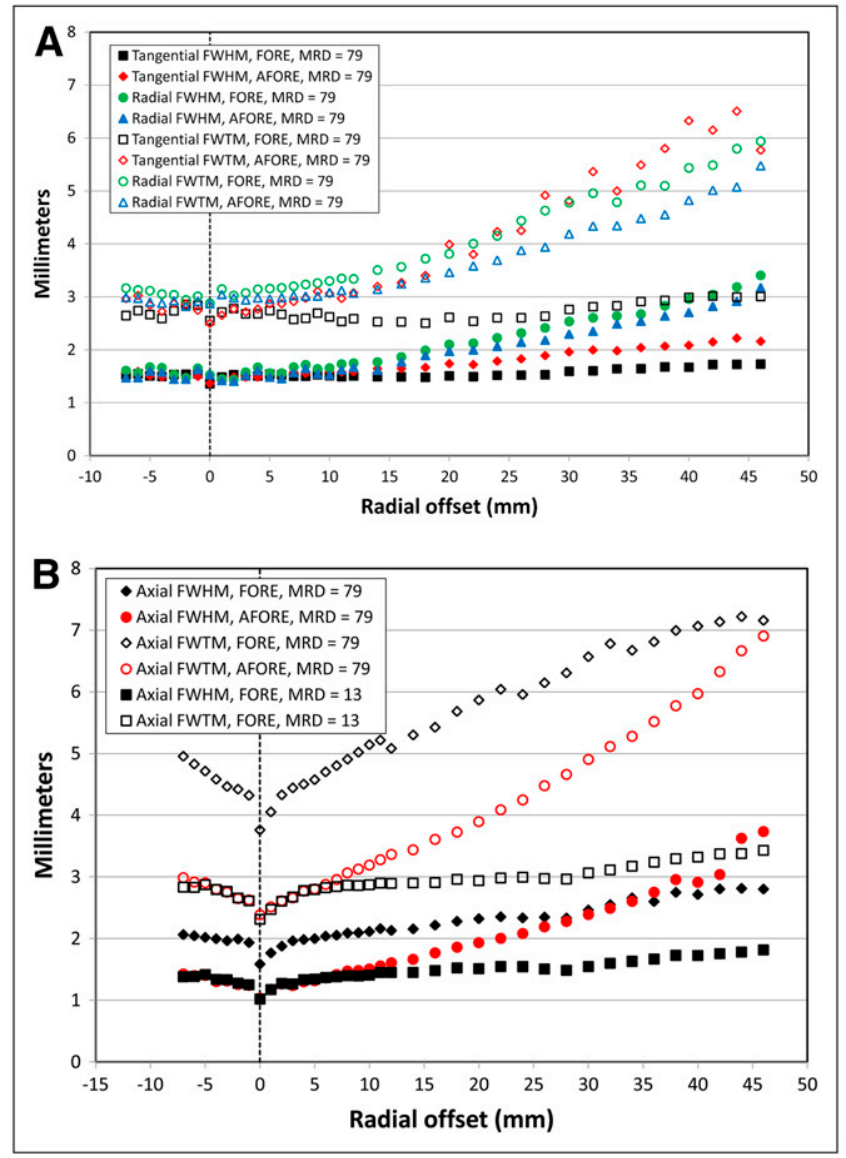

FIGURE 2. (A) Comparison of transaxial spatial resolutions using FORE and AFORE, with FBP as function of radial distance from CFOV. Only results for MRD of 79 have been plotted, because both algorithms yielded negligible differences upon variation of MRD. (B) Comparison of axial spatial resolutions using FORE and AFORE as function of radial distance from CFOV. For AFORE, only results for MRD of 79 have been plotted, because differences upon variation of MRD were negligible. FORE results depended strongly on MRD. As example for small MRDs, FORE results with MRD of 13 are shown.

especially for FWMH. This result is explained by noting that OSEM3D/MAP is a fully 3D reconstruction technique without rebinning errors and that parallax or DOI effects in both the transaxial and the axial directions are suppressed because they have already been modeled in the P-matrices and blur kernels that are used in each iteration step of this reconstruction algorithm. Further, all LORs belonging to an MRD of 79 are being used, so maximum sensitivity is maintained. However, OSEM3D/MAP parameters still require optimization in terms of overall image quality (uniformity, recovery coefficients for small lesions, quantitative imaging, etc.). This optimization was outside the scope of the present study.

The spatial resolution of the Inveon based on FORE and FBP can be compared with that of the commercial crystalbased small-animal scanners given in Table 1. For the F120, Figures $1 \mathrm{~A}$ and $1 \mathrm{~B}$ of Kim et al. (12) demonstrate trans- axial spatial resolutions similar to those for the Inveon. The same holds for the axial resolutions of an MRD of 46 (which is closest to the maximum MRD of 47 of the F120). The Mosaic (Philips) (13) is characterized by FWHMs ranging between 2.7 (radial, CFOV) and $5.8 \mathrm{~mm}$ (axial, EFOV; $46 \mathrm{~mm}$ from radial center), which are larger than the corresponding values for the Inveon. The Vista (GE Healthcare) has an FWHM of $1.4 \mathrm{~mm}$ for all directions in the CFOV. The tangential resolution is more or less independent of the radial offset, whereas both the axial and the radial FWHMs increase to approximately $2.4 \mathrm{~mm}$ for the radial EFOV at $29 \mathrm{~mm}$ (data estimated from Figure $1 \mathrm{~A}$ in Wang et al. (14)). The radial and tangential resolutions of the Vista are approximately equal to those of the Inveon. Axial resolution is better for the Vista than for the Inveon at an MRD of 79; however, for an MRD of 46 (which gives a better comparison with the smaller maximum LOR acceptance angle of $22.1^{\circ}$ of the Vista), axial FWHMs are similar. For the ClearPET (Raytest) in the small-crystal ring configuration (diameter, $135 \mathrm{~mm}$ ), FWHMs in all directions were reported as less than or equal to $1.3 \mathrm{~mm}$ in the CFOV and less than or equal to 2.0 at a radial offset of
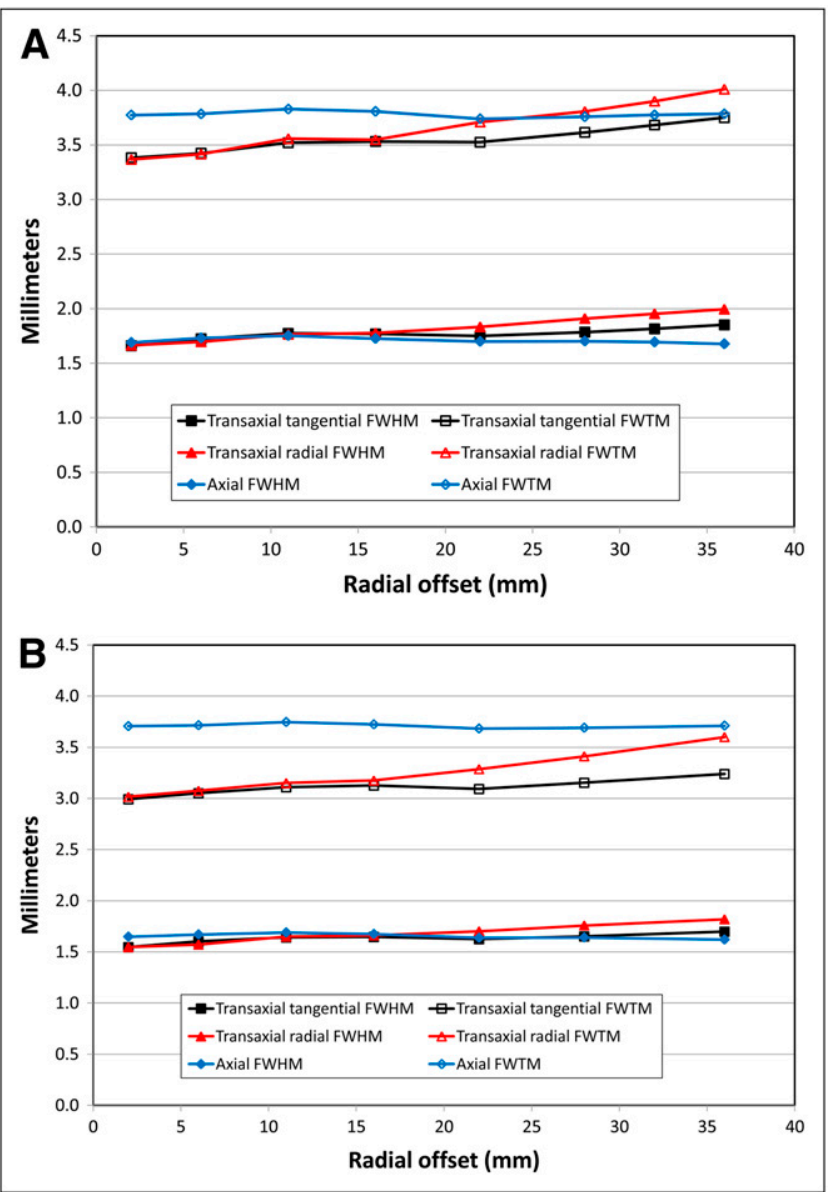

FIGURE 3. Spatial resolution (FWHM and FWTM) in all directions as function of radial distance from CFOV for OSEM3D/MAP with $\beta$ of 1.5 (A) and $\beta$ of $0.5(B)$. 

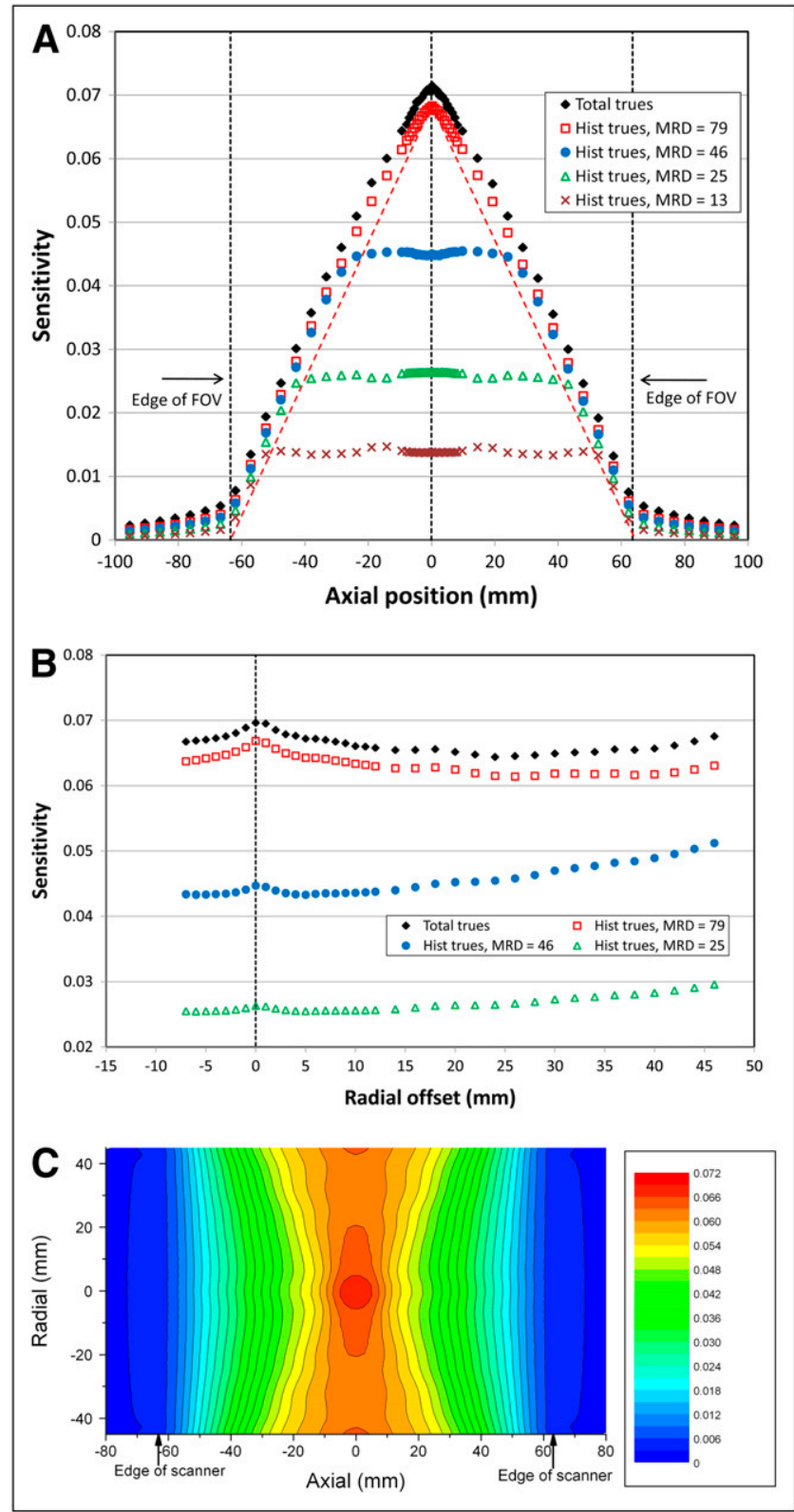

FIGURE 4. (A) Axial sensitivity profiles at radial center for different MRDs. Dashed red line indicates sensitivity as would be obtained for MRD of 79, with linear decrease down to axial edge of FOV of scanner. (B) Radial sensitivity profiles for middle plane for different MRDs. (C) Sensitivity for complete FOV for MRD of 79. All sensitivities correspond to default $\Delta \mathrm{E}$ and $\Delta \mathrm{t}$ and are based on histogrammed (Hist) trues rates, except for $\downarrow$, which were calculated using total trues rate.

$30 \mathrm{~mm}$ from the center (15). These values are clearly better than those of the Inveon. However, unlike for the other scanners, the values have not been obtained using the NEMA prescriptions (9). Instead of using rebinning and FBP, an iterative 3D reconstruction method (OSMAPOSL) was used, and resolutions were determined in gaussian fits of the profiles instead of by using the NEMA interpolation method. On the other hand, when comparing the ClearPET OSMAPOSL results with the Inveon OSEM3D/MAP results, we found the ClearPET performance to be still better. It is not clear yet, though, to what extent this should be attributed to differences in the MAP-based reconstruction algorithms or to real differences in intrinsic spatial resolution between the scanners.

\section{Sensitivity}

The peak sensitivity as specified by the manufacturer $(>0.1)$ is confirmed by our value of 0.111 . This value holds for the CFOV, large $\Delta \mathrm{E}$ and $\Delta \mathrm{t}$, and total trues rate instead of histogrammed trues rate. Sensitivity values for default $\Delta \mathrm{E}$ are lower, as shown in Table 3. The measured sensitivities are almost independent of $\Delta \mathrm{t}$, indicating that the timing resolution is good for the counting rates observed with the present point source activity (prompt rates, 86.0 and $56.5 \mathrm{kcps}$ for $\Delta \mathrm{E}=250-750$ and $350-650 \mathrm{keV}$, respectively).

Because sensitivities were calculated using the rate at which photon pairs are emitted from the ${ }^{22} \mathrm{Na}$ point source, their accuracy is determined by the accuracy of the source activity (the influence of count statistics on the observed trues rate is negligible in view of the large number of coincidence counts in each measurement). This accuracy is $\pm 4 \%$, which leads to $\pm 4 \%$ accuracy of all sensitivity values presented in this article. Our dose calibrators are regularly checked by the manufacturer using sources (provided by North American Scientific Inc.) that have been calibrated against the standards at The Netherlands Metrology Institute (Nederlands Meetinstituut) and the German Federal Metrology Institute (Physikalisch-Technische Bundesanstalt). However, the activity, as specified on the ${ }^{22} \mathrm{Na}$ point source calibration data sheet of the IPL, is $7 \%$ smaller than the value measured by us. Accordingly, when using this value, we would have increased all sensitivity data by $7 \%$. However, we preferred to use the value measured by us because the accuracy specified by IPL is as large as $\pm 15 \%$.

The axial sensitivity profiles in Figure 4A are in accordance with straightforward geometric considerations based on the total number of LORs contained in the FOV of the scanner when the point source is stepped in the axial direction. This explains the central plateaus for an MRD of less than 79 and the more or less linear drop to zero when moving the point source toward the axial EFOVs. It is not immediately clear why the observed drop in sensitivity is less than predicted by mere geometry, but the following could be speculated. For a point source in the CFOV, the maximum angle at which coincidences are detected is $38.3^{\circ}$, whereas for positions closer to the axial edges, this angle decreases. Oblique photons may be less effectively detected by crystals at the edge of detector blocks because of the gaps between blocks, which could explain the observed less-than-linear drop of sensitivity. Coincidences are detected even when the point source is located beyond the EFOVs. This effect should be attributed mostly to scatter effects in the detector crystals or in the lead ring that 
TABLE 3. Peak Sensitivity for Different Energy and Coincidence Time Windows for MRD of 79

\begin{tabular}{lcccc} 
& \multicolumn{5}{c}{$\Delta t(\mathrm{~ns})$} & 4.1 & 4.7 \\
\cline { 2 - 5 }$\Delta \mathrm{E}(\mathrm{keV})$ & 2.8 & 3.4 & $0.068(0.072)$ & $0.068(0.072)$ \\
$350-650$ & $0.068(0.071)$ & $0.068(0.072)$ & $0.101(0.110)$ & $0.101(0.111)$ \\
$250-750$ & $0.099(0.107)$ & $0.100(0.109)$ & & \\
& & &
\end{tabular}

shields the detectors at the axial EFOVs and to a lesser extent to the natural radioactivity of LSO crystals because of the presence of ${ }^{176} \mathrm{Lu}$. The background contribution just outside the FOV at $71 \mathrm{~mm}$ from the center was $6 \%$.

Sensitivity across the whole FOV as shown in Figure 4C may be illustrative for researchers who wish to optimize their experiments by scanning more than 1 animal at the same time. On the basis of only sensitivity, it would be preferable to place 2 animals (e.g., mice) on top of or next to each other instead of behind each other. However, it should be verified whether attenuation and scatter correction is still effective, because more attenuating and scattering mass will then be present in the transaxial FOV.

It is interesting to compare the sensitivity of the Inveon with its predecessor, the F120, which has the same crystal size and similar geometry but fewer crystal rings. A sensitivity of 0.040 was found for an $\Delta \mathrm{E}$ of $350-650 \mathrm{keV}$ (Table 2 in Kim et al. (12)) for the maximum MRD of 47. The closest attainable MRD in the Inveon is 46, for which we found a sensitivity of 0.0451 (average plateau value in Fig. 4A). It is therefore concluded that the higher sensitivity of the Inveon is due not only to its larger axial FOV but also, for approximately $13 \%$, to its better detection efficiency for each crystal ring separately. In fact, the difference is still somewhat larger, because the data of Kim et al. (12) were based on total trues rate (written communication with J.S. Lee, second author of

A
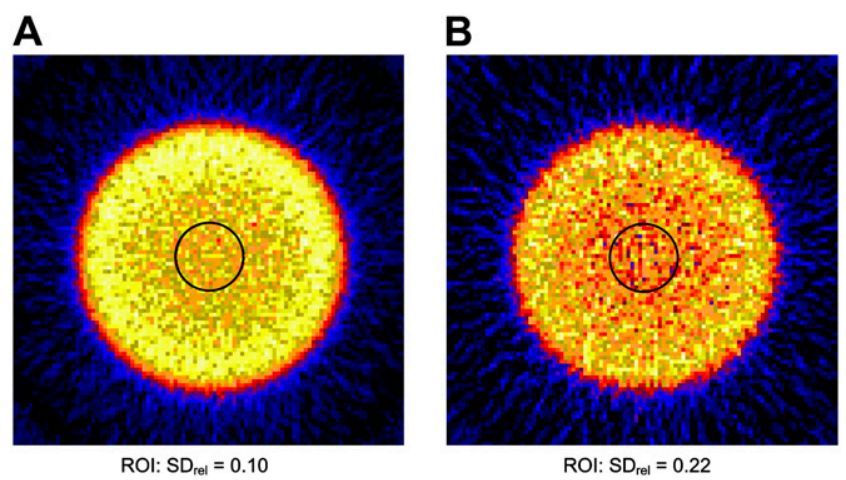

$\mathrm{ROI}: \mathrm{SD}_{\text {rel }}=0.10$

$\mathrm{ROI}: \mathrm{SD}_{\text {rel }}=0.22$

FIGURE 5. Comparison of reconstructed images of uniform ${ }^{68} \mathrm{Ge}$ cylinder with 6 -cm diameter using FORE (A) and AFORE (B) for central transaxial plane. Reconstruction algorithm for both images was FBP with matrix size of $128 \times 128$. Color was scaled to maximum pixel value in both images. $\mathrm{SD}_{\text {rel }}$ was calculated for circular regions of interest. $\mathrm{ROI}=$ region of interest. reference 12,2008 ), whereas we used histogrammed trues rate in our study.

The Inveon showed higher sensitivity than did the other commercial small-animal scanners presented in Table 1. The values reported in the literature for these scanners cannot directly be compared with those of the Inveon, mainly because of the use of different energy windows, but they are summarized here. The peak sensitivity of the Mosaic for an $\Delta \mathrm{E}$ of $410-665 \mathrm{keV}$ is 0.0114 (13). The Vista is characterized by peak sensitivities of $0.065,0.040$, and 0.021 for energy windows of $100-700,250-700$, and 400$700 \mathrm{keV}$, respectively (14). Finally, the reported peak sensitivity for the ClearPET is 0.045 (15) for the small-diameter, full-ring configuration with an $\Delta \mathrm{E}$ of $250-750 \mathrm{keV}$.

\section{CONCLUSION}

The Inveon small-animal PET scanner is characterized by high spatial resolution and high sensitivity. However, when using rebinning and 2D reconstruction, a trade-off occurs between these 2 parameters. Axial spatial resolution is deteriorated by both the axial parallax or DOI effect and the breakdown of FORE for large LOR acceptance angles. The way to retain both high sensitivity and high spatial resolution in all directions is by using fully $3 \mathrm{D}$ image reconstruction algorithms such as OSEM3D/MAP, which form part of the standard scanner software.

Although the spatial resolution performance of the Inveon is similar to that of scanners such as the F120 or the Vista (results for the ClearPET cannot properly be compared because of different methodology), the peak sensitivity of 0.111 , obtained with an $\Delta \mathrm{E}$ of $250-750 \mathrm{keV}$, is presently unrivaled.

\section{REFERENCES}

1. Newport DF, Siegel SB, Swann BK, et al. QuickSilver: a flexible, extensible, and high-speed architecture for multi-modality imaging. IEEE Nucl Sci Symp Conf Rec 2006. 2006;4:2333-2334.

2. Atkins BE, Pressley DR, Lenox MW, Swann BK, Newport DF, Siegel SB. A data acquisition, event processing and coincidence determination module for a distributed parallel processing architecture for PET and SPECT imaging. IEEE Nucl Sci Symp Conf Rec 2006. 2006;4:2439-2442.

3. McFarland AR, Newport DF, Atkins B, Pressley D, Siegel S, Lenox MA. A compact PCI based event routing subsystem for PET and SPECT data acquisition. IEEE Nucl Sci Symp Conf Rec 2006. 2006;5:3091-3093.

4. McFarland AR, Siegel S, Newport DF, Mintzer R, Atkins B, Lenox M. Continuously sampled digital pulse processing for Inveon small animal PET scanner. IEEE Nucl Sci Symp Conf Rec 2007. 2007;6:4262-4265. 
5. Mintzer RA, Siegel SB. Design and performance of a new pixelated LSO/ PSPMT gamma-ray detector for high resolution PET imaging. IEEE Nucl Sci Symp Conf Rec 2007. 2007;5:3418-3422.

6. Defrise M, Kinahan PE, Townsend DW, Michel C, Sibomana M, Newport DF. Exact and approximate rebinning algorithms for 3-D PET data. IEEE Trans Med Imaging. 1997;16:145-158.

7. Cherry SR, Sorenson JA, Phelps ME. Physics in Nuclear Medicine. Philadelphia, PA: Saunders/Elsevier Science; 2003.

8. Matej S, Karp JS, Lewitt RM, Becher AJ. Performance of the Fourier rebinning algorithm for PET with large acceptance angles. Phys Med Biol. 1998;43:787795.

9. National Electrical Manufacturers Association. NEMA Standards Publication NU 2-2007. Performance Measurements of Positron Emission Tomographs. Rosslyn, VA: National Electrical Manufacturers Association; 2007:8-9.

10. Fahey FH. Data acquisition in PET imaging. J Nucl Med Technol. 2002;30:39-49.
11. Stabin MG, da Luz LC. Decay data for internal and external dose assessment. Health Phys. 2002;83:471-475.

12. Kim JS, Lee JS, Im KC, et al. Performance measurement of the microPET focus 120 scanner. J Nucl Med. 2007;48:1527-1535.

13. Huisman MC, Reder S, Weber AW, Ziegler SI, Schwaiger M. Performance evaluation of the Philips MOSAIC small animal PET scanner. Eur J Nucl Med Mol Imaging. 2007;34:532-540.

14. Wang Y, Seidel J, Tsui BM, Vaquero JJ, Pomper MG. Performance evaluation of the GE Healthcare eXplore VISTA dual-ring small-animal PET scanner. J Nucl Med. 2006;47:1891-1900.

15. Roldan PS, Canadas M, Dietzel O, Pautrot C, Sarasola I, Wagner A. Performance evaluation of Raytest ClearPET, a PET scanner for small and medium size animals. IEEE Nucl Sci Symp Conf Rec 2007. 2007;4:2859-2864.

16. Roldan PS, Chereul E, Dietzel O, et al. Raytest ClearPET (TM): a new generation small animal PET scanner. Nucl Instrum Methods Phys Res A. 2007; 571:498-501. 